\title{
Assessment of the groundwater quantitative status in the Aosta Plain (North-West Italy): applicability of the national guidelines defined by ISPRA
}

\section{Valutazione dello stato quantitativo delle acque sotterranee della piana d'Aosta: applicabilità delle linee guida ISPRA}

Gennaro A. Stefania, Michele Bigoni, Chiara Zanotti, Marco Rotiroti, Fulvio Simonetto, Pietro Capodaglio, Letizia Fumagalli, Tullia Bonomi

Riassunto: La direttiva quadro sulle acque dell'Unione Europea, recepita dall'ordinamento nazionale, richiede agli Stati Membri di valutare lo stato quantitativo e qualitativo dei corpi idrici sotterranei e superficiali e di raggiungerne il "buono" stato per tutti i corpi idrici entro il 2027. Il presente studio è stato sviluppato per determinare ai sensi della vigente normativa, tramite

Keywords: Aosta Valley, aquifer, quantitative status, trend,
water balance.
$\begin{aligned} & \text { Parole chiave: Valle d'Aosta, acquifero, stato quantitativo, } \\ & \text { tendenza, bilancio idrico. }\end{aligned}$

\section{Marco ROTIROTI 棒: \\ Dipartimento di Scienze dell'Ambiente e della Terra \\ Università degli Studi di Milano-Bicocca, Milano, Italia. \\ marco.rotiroti@unimib.it}

\section{Gennaro A. STEFANIA}

Dipartimento di Scienze dell'Ambiente e della Terra

Università degli Studi di Milano-Bicocca, Milano, Italia.

\section{Michele BIGONI}

Dipartimento di Scienze dell'Ambiente e della Terra

Università degli Studi di Milano-Bicocca, Milano, Italia.

\section{Chiara ZANOTTI}

Dipartimento di Scienze dell'Ambiente e della Terra

Università degli Studi di Milano-Bicocca, Milano, Italia.

\section{Fulvio SIMONETTO}

Agenzia Regionale per la Protezione dell'Ambiente della Valle d'Aosta

St. Christophe (AO), Italia.

Pietro CAPODAGLIO

Agenzia Regionale per la Protezione dell'Ambiente della Valle d'Aosta St. Christophe (AO), Italia.

\section{Letizia FUMAGALLI}

Dipartimento di Scienze dell'Ambiente e della Terra

Università degli Studi di Milano-Bicocca, Milano, Italia.

Tullia BONOMI

Dipartimento di Scienze dell'Ambiente e della Terra

Università degli Studi di Milano-Bicocca, Milano, Italia.

Ricevuto/Received: 19 December 2019-Accettato/Accepted: 12 March 2020 Pubblicato online/Published online: 30 June 2020

This is an open access article under the CC BY-NC-ND license:

http://creativecommons.org/licenses/by-nc-nd/4.0/

(C) Associazione Acque Sotterranee 2020 applicazione di apposite linee guida ISPRA, lo stato quantitativo delle acque sotterranee della piana d'Aosta al fine di supportare gli Enti Locali preposti alla periodica valutazione dello stato dei corpi idrici. Nell'area di studio, durante il periodo 2000-2017, i livelli piezometrici sono stati misurati su un totale di 213 pozzi/piezometri. Applicando i quattro test previsti dalle suddette linee guida, lo stato quantitativo è stato valutato in funzione delle variazioni pluriennali del livello piezometrico e del bilancio idrico, delle acque superficiali connesse e degli ecosistemi terrestri dipendenti. Si è giunti ad una valutazione di "buono" stato quantitativo, ma non senza difficoltà operative. Lapplicazione delle procedure proposte dalle linee guida ha infatti permesso di evidenziare le criticità relative alla rete di monitoraggio dell'area di studio che ostacolano lo svolgimento delle valutazioni, in particolare per quanto riguarda la numerosità, la distribuzione spaziale e i periodi di riferimento delle serie di dati. Sono quindi state elaborate indicazioni a supporto del monitoraggio per migliorare la qualità dei dati e favorire le future determinazioni dello stato quantitativo.

\begin{abstract}
The EU Water Framework Directive requires Member States to assess the quantitative and qualitative status of groundwater bodies and to achieve a good status by 2027. The present study was developed in order to assess the groundwater quantitative status in the Aosta Plain (NW Italy), following the methodology proposed through a guideline defined by the Italian Institute for Environmental Protection and Research (ISPRA), with the aim of supporting local authorities in their periodic assessment of water bodies status. In the study area, during the period 2000-2017, groundwater levels were measured in 213 wells and piezometers. Performing the four tests required by the abovementioned guideline, the quantitative status was assessed on the basis of multi-year groundwater level variations, water balance and the interconnections of groundwater with surface water bodies and groundwaterdependent ecosystems. A good status was assessed for the study area, although some critical aspects emerged from the application of the guideline methodology: the data collected by the monitoring network are not sufficiently robust in terms of sample size, spatial distribution, temporal interval and frequency to fulfill the requirements of the guideline methodology. Therefore, some recommendations to improve the data quality and better address the future assessment of groundwater quantitative status were given.
\end{abstract}




\section{Introduzione}

Con l'emanazione della Water Framework Directive (WFD; EC 2000) e della Groundwater Directive (GWD; EC 2006), sono state poste le basi per una gestione uniforme e condivisa delle acque a livello europeo. Queste direttive forniscono un inquadramento tecnico e normativo rivolto agli Stati Membri, affinché sviluppino metodologie dedicate nell'ambito della protezione e gestione delle risorse idriche, sia sotterranee che superficiali. In Italia, la normativa nazionale (D. Lgs. 30/09) che ha recepito le suddette direttive europee non fornisce però precise indicazioni metodologiche sulla valutazione dello stato quantitativo delle risorse idriche sotterranee. Al fine di colmare questa lacuna, l'Istituto Superiore per la Protezione e la Ricerca Ambientale (ISPRA) ha sviluppato nel 2016, con il contributo delle Agenzie Regionali per la Protezione Ambientale (ARPA), delle linee guida dedicate (Percopo et al. 2017). Scopo di tale documento è quello di fornire un quadro standardizzato a livello nazionale per una valutazione scientificamente robusta dello stato quantitativo e per la programmazione del monitoraggio dei corpi idrici sotterranei. Le linee guida raccolgono gli strumenti tecnici necessari alla valutazione dello stato delle acque sotterranee, con l'obiettivo di omogeneizzare le metodologie e far sì che i risultati siano rappresentativi delle singole realtà regionali e confrontabili a livello nazionale. Il punto di forza di questa metodologia consiste in una valutazione olistica dello stato delle acque sotterranee, estendendo l'analisi alle matrici ambientali direttamente o indirettamente connesse ad esse, permettendo un più esteso livello di tutela. Affinché l'efficacia delle linee guida possa essere rafforzata ed il loro utilizzo entrare a regime, queste beneficiano dell'applicazione a casi studio reali per metterne in luce gli aspetti positivi e le eventuali criticità. In questo contesto si inserisce il presente lavoro, i cui obiettivi sono:

- valutare lo stato quantitativo delle acque sotterranee della piana di Aosta con una metodologia condivisa e scientificamente robusta, tramite applicazione delle linee guida ISPRA;

- individuare eventuali criticità nell'applicazione delle linee guida;

- fornire supporto e indicazioni agli Enti Locali dell'area di studio per lo sviluppo delle valutazioni future di stato quantitativo.

\section{Materiali e metodi \\ Area di studio \\ Inquadramento geografico}

L'area oggetto dello studio è la piana d'Aosta, che si estende longitudinalmente in direzione O-E all'interno della Regione Autonoma della Valle d'Aosta tra Villeneuve e Pontey (Fig. 1). Sviluppandosi con una lunghezza di circa $35 \mathrm{~km}$ ed ampiezza massima di $2 \mathrm{~km}$ presso il capoluogo, la piana d'Aosta costituisce il fondovalle principale della regione, con quote che variano tra $700 \mathrm{~m}$ sul livello del mare (m s.l.m.), sui versanti occidentali, e $460 \mathrm{~m}$ s.l.m., nel settore orientale. La piana è compresa nel bacino idrografico della Dora Baltea, che scorre in direzione $\mathrm{O}-\mathrm{E}$, con decise deviazioni determinate dai conoidi di entrambi i versanti, e riceve acqua da numerosi tributari.
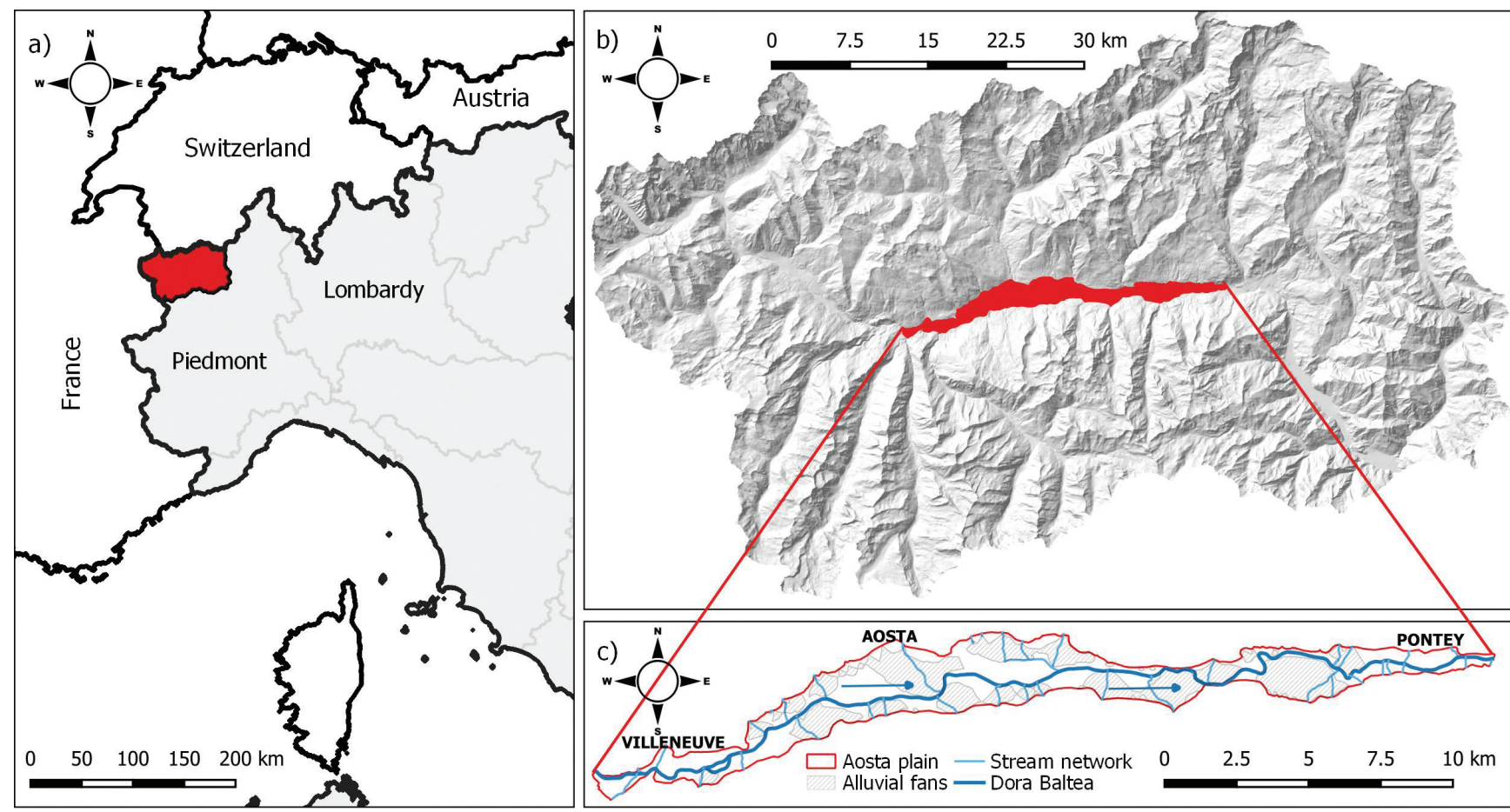

Fig. 1 - a) Ubicazione della regione Valle d'Aosta (in rosso); b) Ubicazione della piana d'Aosta (in rosso); c) Dettaglio della piana d'Aosta.

Fig. 1 - a) Location of Aosta Valley Region (in red). b) Location of the Aosta Plain (in red). c) Detailed view of the Aosta Plain. 


\section{Inquadramento idrogeologico}

La piana ospita un sistema acquifero formato da depositi alluvionali, fluvioglaciali, lacustri e di conoide. La tessitura dei depositi che costituiscono gli acquiferi varia da ghiaiosa a sabbiosa, con locali e discontinue intercalazioni argillose. Il sistema acquifero esistente è schematizzabile come segue (Fig. 2; Capodaglio and Simonetto 2017a; Stefania et al. 2018a, 2019):

- è presente un acquifero superficiale di tipo libero, sfruttato da prelievi per diversi scopi (potabile, industriale, domestico, ecc.), con uno spessore che varia da $50 \mathrm{~m}$ (nella parte orientale) a $90 \mathrm{~m}$ (nella parte occidentale); questo acquifero localmente, nella parte orientale, è a sua volta suddiviso in una parte libera ed una sottostante parte semi-confinata dalla presenza di un acquitardo limosoargilloso, con spessore di circa $5 \mathrm{~m}$, che si trova alla profondità di circa $20 \mathrm{~m}$ dal piano campagna (Bonomi et al. 2015a; Rotiroti et al. 2015a; Triganon et al. 2003);
- è inoltre presente un acquifero profondo di tipo confinato, non sfruttato da prelievi né ad oggi raggiunto da perforazioni, che ha il tetto ad una profondità dal piano campagna che varia da circa $90 \mathrm{~m}$, nella parte orientale, a $140 \mathrm{~m}$, nella parte occidentale (Stefania et al. 2018b) ed il letto ad una profondità attualmente non nota;

- la separazione tra questi due acquiferi è definita da un acquitardo argilloso-limoso di circa $40 \mathrm{~m}$ di spessore, che presenta però delle discontinuità nella zona centroorientale dell'area di studio, dove i due acquiferi sono, localmente, in contatto (Stefania et al. 2018b).

La falda scorre principalmente in direzione O-E con un gradiente piuttosto regolare (0.4-0.5\%; Bonomi et al. 2013), con variazioni stagionali che presentano un minimo invernale/ primaverile e un massimo estivo (Bonomi et al. 2015b). Le variazioni stagionali sono visibili in Fig. 2, che riporta due mappe piezometriche, estratte dal modello numerico di flusso elaborato da Stefania et al. (2018b), per le condizioni di
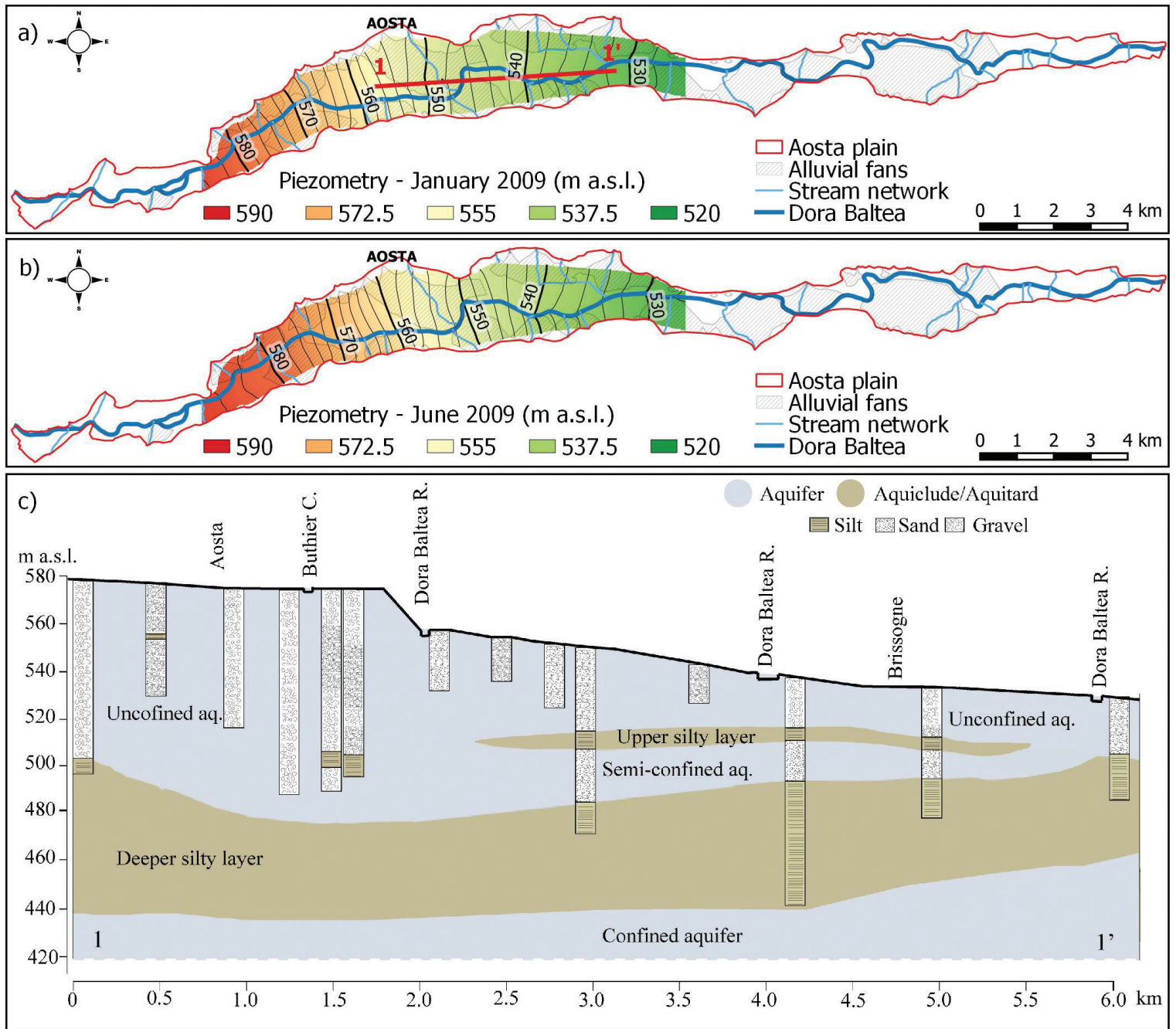

Fig. 2 - a) Mappa piezometrica nelle condizioni di minimo (gennaio) per l'acquifero superficiale risultante dal modello di flusso elaborato da Stefania et al. 2018b. b) Mappa piezometrica nelle condizioni di massimo (giugno) per l'acquifero superficiale risultante dal modello di flusso elaborato da Stefania et al. 2018 b. c) Sezione schematica dell'acquifero (modificato da Stefania et al. 2019).

Fig. 2 - a) Piezometric map referred to January 2009 (representative of the minimum conditions) for the shallow aquifer resulted from the groundwater flow model by Stefania et al. 2018b. b) Piezometric map referred to June 2009 (representative of the maximum conditions) for the shallow aquifer resulted from the groundwater flow model by Stefania et al. 2018b. c) Schematic cross-section of the aquifer system (modified from Stefania et al. 2019). 
minimo e massimo. Questa escursione stagionale è maggiore nelle aree occidentali della piana, con fluttuazioni superiori ai $5 \mathrm{~m}$, e decresce progressivamente verso est, fino a raggiungere valori di circa $1 \mathrm{~m}$ nella zona centro-orientale.

La dinamica di falda suggerisce una progressiva variazione dei rapporti falda-fiume procedendo da monte, dove il fiume Dora Baltea alimenta la falda, verso valle, dove il fiume in prevalenza drena la falda (Bonomi et al. 2013; Stefania et al. 2018b; Tiwari et al. 2017); tuttavia esistono evidenze di una probabile inversione stagionale del rapporto di alimentazione e drenaggio nell'area ad est di Aosta (Stefania et al. 2018b).

Gli afflussi al sistema idrico sotterraneo sono determinati principalmente dagli scioglimenti nivali, dalle precipitazioni e dalle alimentazioni dei corpi idrici superficiali, mentre i deflussi principali dal sistema sono dovuti ai prelievi da pozzo (principalmente ad uso potabile ed industriale) e all'effetto drenante esercitato dai corpi idrici superficiali.

\section{Valutazione semplificata dello stato quantitativo}

Prima dell'introduzione di precise indicazioni normative e delle linee guida ISPRA, la classificazione dello stato quantitativo dell'acquifero in esame era effettuata esaminando la regressione lineare calcolata sulle serie storiche piezometriche pluriennali, laddove disponibili.

L'esame complessivo di questi dati ha consentito in passato di definire lo stato quantitativo senzaltro "buono" (Capodaglio and Simonetto 2017b), non rilevandosi in alcun caso sul lungo periodo abbassamenti significativi. È bene tuttavia evidenziare che in ogni caso, ai sensi della normativa (D. Lgs. 30/09), un'eventuale classificazione in stato "scarso" dovrebbe essere ascrivibile ad abbassamenti determinati da pressioni antropiche (ad es. prelievi eccessivi) e non a cause naturali (ad es. ridotta ricarica). Uno degli scopi delle procedure ISPRA è appunto quello di considerare anche tali variabili, e ciò viene affrontato nel presente studio.

\section{Descrizione della procedura ISPRA}

Le linee guida ISPRA (Percopo et al. 2017) propongono una procedura strutturata in quattro test successivi, ognuno dei quali mira a valutare un aspetto specifico della falda: bilancio idrico (Test 1), acque superficiali connesse (Test 2), ecosistemi terrestri dipendenti (Test 3) e intrusione salina (Test 4). Ogni test ha due esiti possibili, "stato buono" o "stato scarso", e seguono un criterio "one out, all out", per cui il fallimento di anche solo uno di essi comporta l'attribuzione del giudizio di stato quantitativo "scarso" all'intero corpo idrico sotterraneo.

\section{Test 1 - Bilancio idrico}

Questo test mira a valutare l'equilibrio tra i prelievi medi sul lungo termine e le risorse idriche naturali disponibili, al netto dei volumi necessari al mantenimento del "buono" stato degli ecosistemi terrestri dipendenti dalle acque sotterranee e dei corpi idrici superficiali connessi alla falda. Per questo scopo, l'analisi si compone delle seguenti fasi (Fig. 3):

i. Fase 0 - verifica della presenza di prelievi, in mancanza dei quali lo stato è "buono" per definizione (EC 2000);

ii. Fase A - verifica dell'esistenza di un depauperamento della risorsa riscontrabile come tendenza decrescente statisticamente rilevante dei livelli di falda, verificata con il test di Mann-Kendall;

iii. Fase B - analisi di bilancio idrico tra le risorse naturalmente rinnovabili ed il loro consumo dovuto ai prelievi.

Nel caso in cui nella Fase A emerga una generale stabilità dei trend piezometrici sul lungo periodo, è necessario affrontare la Fase B. Questa fase prevede di valutare se le risorse idriche disponibili, denominate come Available Groundwater Resources (AGR), siano maggiori dei prelievi medi sul lungo periodo (Long-Term Annual Average Abstraction, LTAAQ) (Fig. 3). L'AGR è definita come differenza tra ricarica naturale

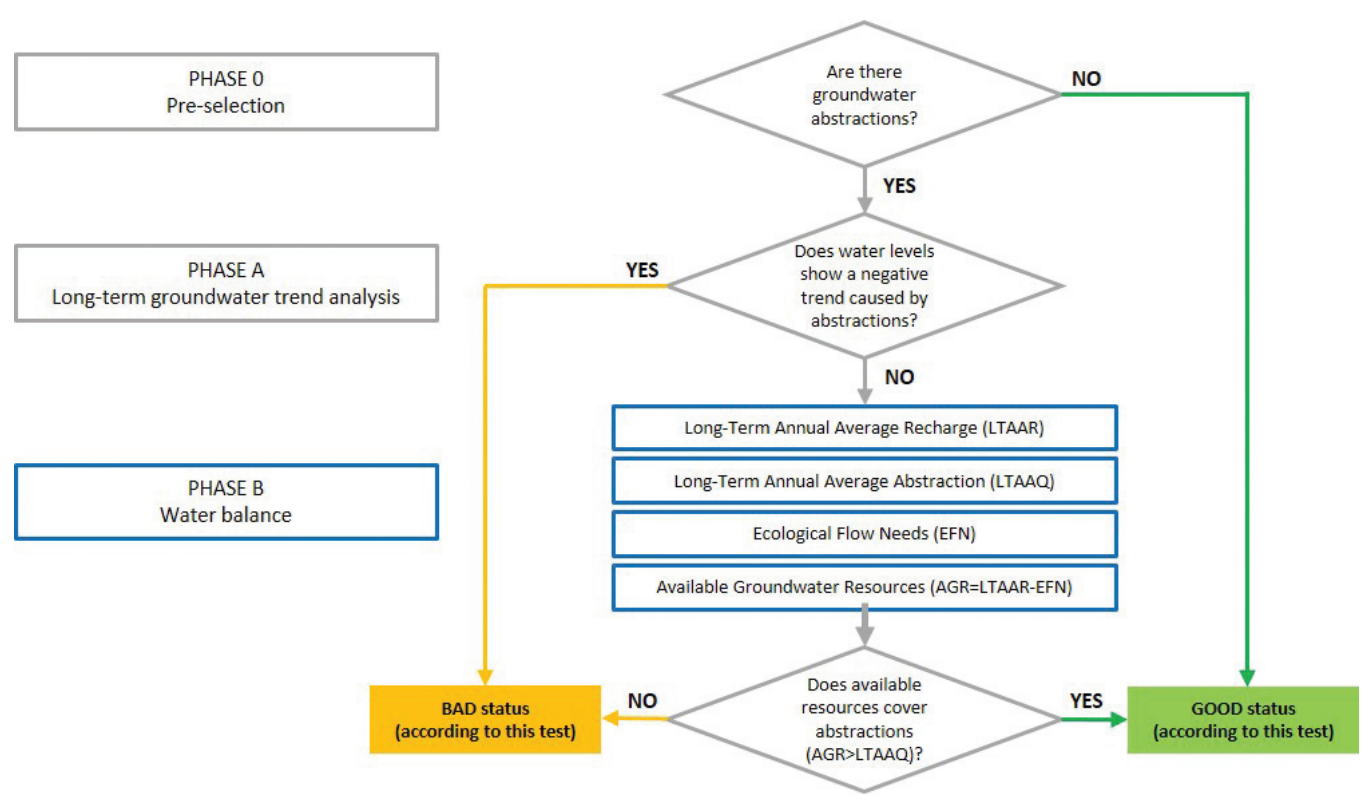

Fig. 3 - Diagramma di flusso per il Test 1 (modificato da EC 2009).

Fig. 3 - Test 1 flowchart (modified from EC 2009). 
sul lungo periodo (Long-Term Annual Average Recharge, LTAAR) e la portata media di falda (sul lungo periodo) che alimenta i corpi idrici superficiali e ne mantiene il "buono" stato ecologico e chimico-fisico (Ecological Flow Needs, EFN), secondo l'equazione:

$$
A G R=L T A A R-E F N
$$

Se $\quad \frac{L T A A Q}{A G R}<1$

allora le risorse idriche sono superiori ai prelievi e lo stato quantitativo può essere considerato "buono", in caso contrario lo stato quantitativo è considerato "scarso".

Per lo svolgimento di questo test la linea guida ISPRA suggerisce l'utilizzo di serie storiche con lunghezza minima di 10-15 anni e con frequenza minima di campionamento mensile. Nei casi in cui queste condizioni non possano essere soddisfatte, sono ritenute comunque accettabili serie storiche non inferiori a 6 anni recenti e costituite da un numero di misure/anno non inferiore a 2 , comunque riferibili agli estremi annuali (ovvero 1 misura per il minimo piezometrico e 1 misura per il massimo). Questa è la condizione minima accettabile per ottenere un periodo idrologico rappresentativo del sistema indagato, non influenzato dai naturali cicli aridi e umidi (Boni et al. 1993; Huh et al. 2005; Percopo et al. 2017)

\section{Test 2 - Acque superficiali connesse}

Questo test si basa sul concetto di interconnessione tra acque superficiali ed acque sotterranee, riconoscendone la reciproca responsabilità nel mantenimento delle rispettive condizioni quali-quantitative (Winter et al. 1998). Lo stato quantitativo è "scarso" se, a causa di variazioni antropiche dei livelli o delle direzioni di flusso, le acque sotterranee danneggiano la qualità o la quantità delle acque superficiali che da esse dipendono (EC 2000, 2006). Questa procedura si applica solo nel caso in cui i corpi idrici superficiali siano effettivamente connessi al corpo idrico sotterraneo e non siano in "buono" stato chimico o ecologico, definito ai sensi del DM 260/2010.

\section{Test 3 - Ecosistemi terrestri dipendenti}

Gli ecosistemi terrestri possono intrattenere stretti rapporti con le acque sotterranee ed il loro stato di conservazione può dipendere fortemente dall'apporto idrico proveniente dalla falda (Brunke et al. 1997; Hatton et al. 1998). Per questo motivo le linee guida forniscono una procedura dedicata alla valutazione dei potenziali danni cagionati agli ecosistemi. La metodologia permette, in presenza di ecosistemi a rischio o danneggiati e dipendenti dal corpo idrico sotterraneo, di definire lo stato quantitativo "scarso" nel caso in cui il cattivo stato di conservazione sia dovuto ad un'alterazione antropica dei livelli della falda (EC 2011).

\section{Test 4 - Intrusione salina}

In base a questo test, lo stato quantitativo è definito "buono" quando non si verifica intrusione salina, o di altro tipo (ad esempio acque qualitativamente inferiori), nella falda causata da variazioni nelle direzioni di flusso delle acque sotterranee dovute ai prelievi e tali da pregiudicare la qualità delle acque.

\section{Dati disponibili ed elaborazione effettuata \\ Piezometria}

Attraverso il database TANGRAM (Bonomi et al. 2014) è stato possibile considerare $\mathrm{i}$ dati di piezometria riferiti al periodo 2000-2017 provenienti da 213 stazioni di misura, tra pozzi (n. 79) e piezometri (n. 134) noti ad ARPA Valle d'Aosta nella piana (Fig. 4), ottenendo un dataset di 11552 misure.

La Fig. 4 evidenzia i piezometri afferenti alla rete di monitoraggio ARPA Valle d'Aosta, attualmente costituita da n. 5 punti misurati con frequenza mensile e n. 9 punti misurati con frequenza trimestrale, mentre altri n. 20 punti sono misurati con frequenza almeno annuale.

Organizzando $i$ dati in funzione della distribuzione spaziale e temporale è risultata evidente una disomogeneità della frequenza di misura della piezometria nell'area. Questa disomogeneità è causata principalmente dai punti non afferenti alla rete di monitoraggio principale, che presentano misure perlopiù occasionali (Fig. 5), tali da non soddisfare le condizioni proposte dalle linee guida di un minimo di 2 misure all'anno per 6 anni recenti (Fig. 6).

A tale proposito va considerato che il monitoraggio di ARPA Valle d'Aosta è iniziato sistematicamente, ai sensi della normativa all'epoca vigente (D. Lgs. 152/99), nell'anno 2003 e che molti piezometri sono stati perforati solo negli anni successivi. In alcuni casi, inoltre, le misure sono state interrotte a seguito della sopraggiunta inaccessibilità (per cementazione, distruzione accidentale, ecc.) della stazione.

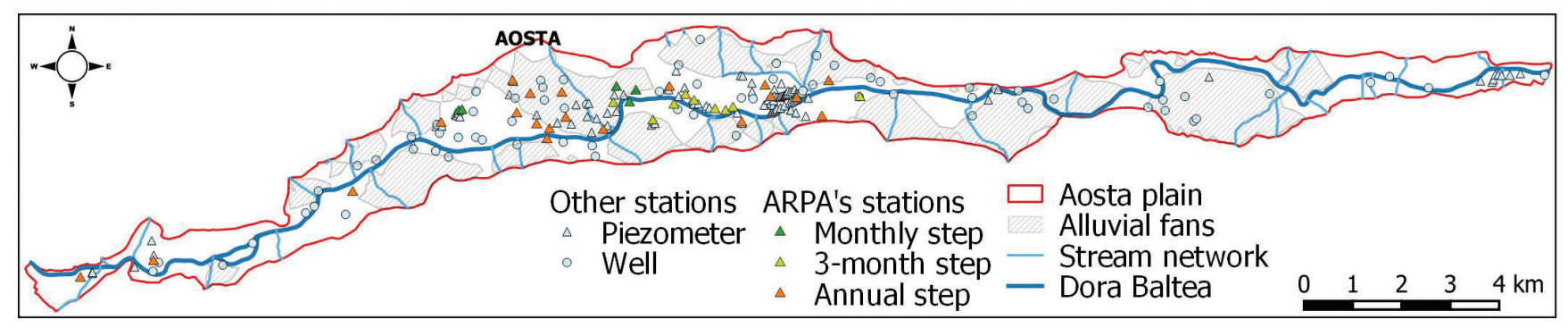

Fig. 4 - Totale delle stazioni di monitoraggio presenti nella piana d'Aosta (213 pozzi/piezometri).

Fig. 4 - Monitoring stations available in the Aosta Plain (213 wells/piezometers). 

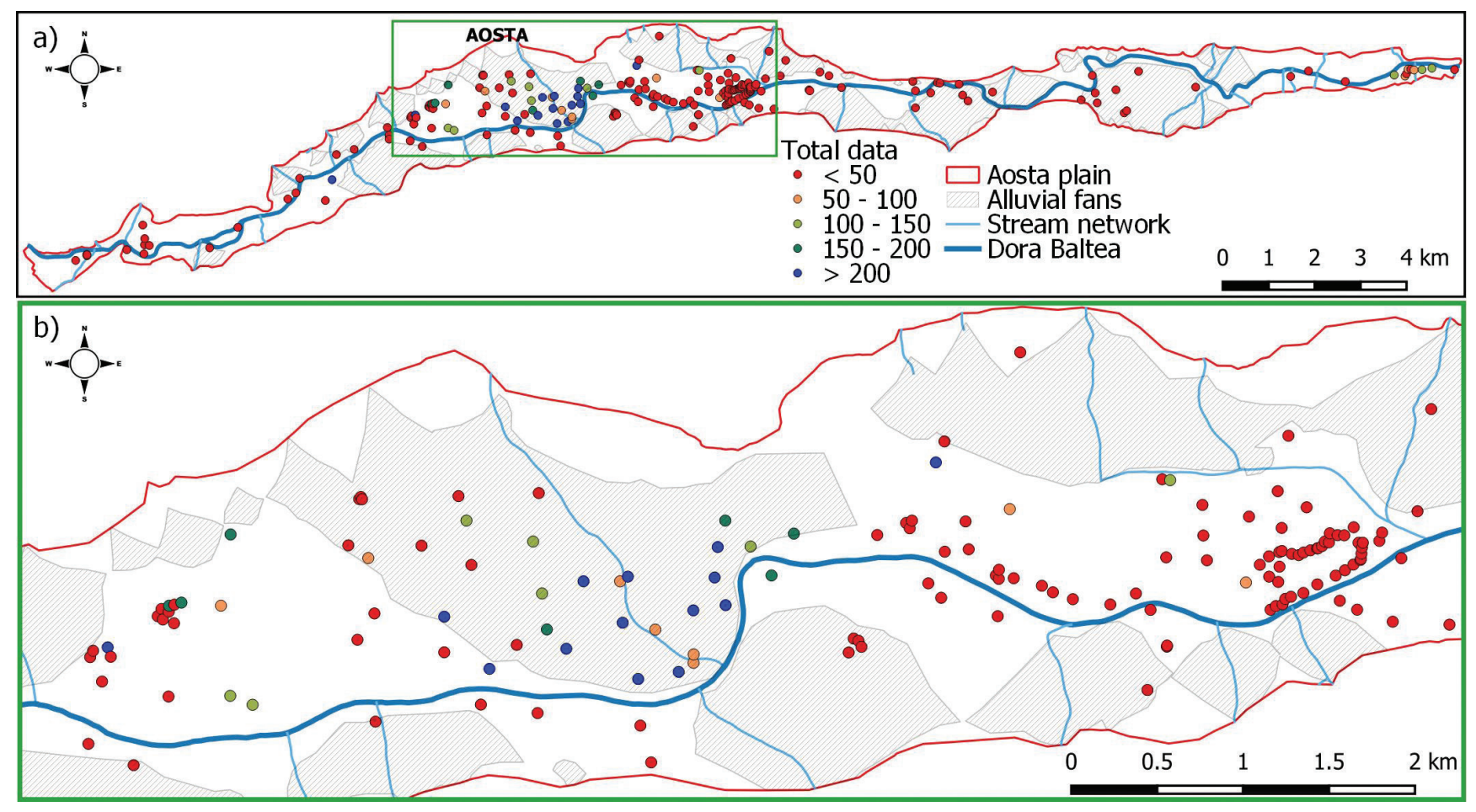

Fig. 5 - a) Abbondanza di dati della rete di monitoraggio nel periodo 2000-2017. b) Dettaglio dell'area centrale della piana.

Fig. 5 - a) Monitoring network data observation counting during the 2000-2017 period. b) Detail of the central area of the plain.

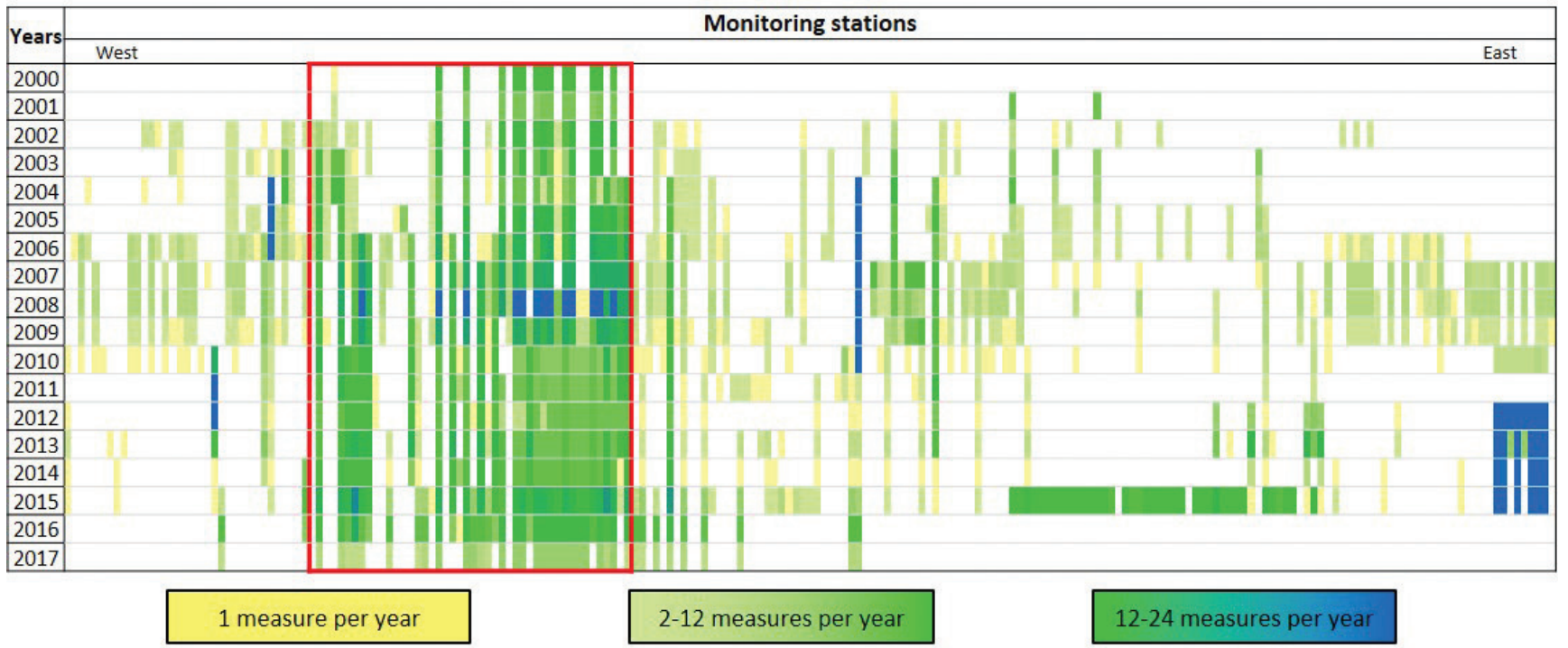

Fig. 6 - Composizione temporale delle serie ordinate da ovest ad est. Il rettangolo rosso indica le stazioni di monitoraggio presenti nell'area urbana di Aosta.

Fig. 6 - Time composition of data series ordered from west to east. The red rectangle indicates the monitoring stations located in the urban area of the town of Aosta.

Date queste condizioni, applicando rigorosamente le indicazioni delle linee guida non sarebbe stato possibile giungere ad una valutazione rappresentativa dell'intera piana. Per realizzare un'analisi più estensiva si è quindi ritenuto di utilizzare anche le serie parziali di durata non inferiore ai 4 anni, non necessariamente fino al presente, forzando l'applicabilità del test di Mann-Kendall anche in presenza di dati mancanti (Braca et al. 2013; Guerra et al. 2017). Le nuove condizioni hanno permesso di selezionare un dataset costituito da 64 punti distribuiti lungo la piana, ad eccezione dell'area compresa tra Brissogne e Chambave (Fig. 7). 


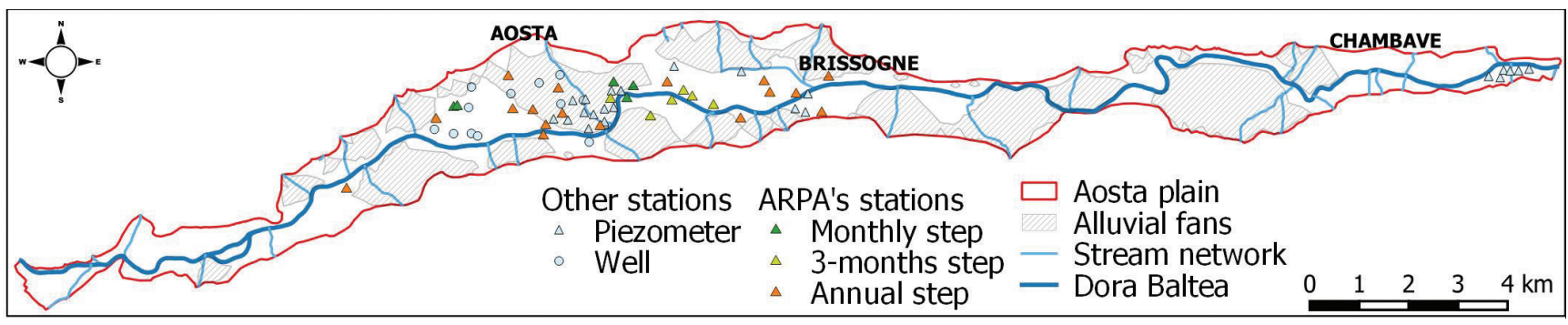

Fig. 7 - Stazioni di monitoraggio selezionate (64 pozzi/piezometri).

Fig. 7 - Selected monitoring stations (64 wells/piezometers).

\section{Analisi delle tendenze pluriennali puntuali}

La fase $A$ del Test 1 è stata affrontata realizzando i grafici piezometria-tempo e le rette di regressione di ogni serie storica per ciascuno dei n. 64 suddetti pozzi/piezometri utilizzando le medie mensili. L'utilizzo della media mensile ha permesso di ridurre l'alterazione della retta di regressione dovuta alla disomogeneità nella distribuzione temporale dei dati, che avrebbe portato a sovrastimare o sottostimare le tendenze, come ad esempio è mostrato in Figura 8 dove per un periodo limitato (da ottobre 2004 ad agosto 2006) sono stati utilizzati data-logger con frequenza di campionamento maggiore rispetto al resto della serie. La maggior densità di dati esercita un peso maggiore sulla regressione, facendo ottenere una pendenza alterata.

La tendenza pluriennale è stata ottenuta applicando il test non parametrico di Mann-Kendall (Mann 1945; Yue and Pilon 2004) con significatività al 5\% per mezzo del software ProUCL 5.1 (U.S. EPA 2015). Questo test, che non necessita di una distribuzione particolare dei dati ed è utilizzabile anche con dati mancanti, confronta ogni dato di una serie con ognuno di quelli che lo seguono, costruendo l'indice $\mathrm{S}$, per verificare se la tendenza ascendente o discendente sia statisticamente significativa:

$$
S=\sum_{i=1}^{n-1} \sum_{j=i+1}^{n} \operatorname{sgn}\left(x_{i}-x_{j}\right)
$$

dove:

$$
\operatorname{sgn}\left(x_{i}-x_{j}\right)=\left\{\begin{array}{l}
+1 \text { se } x_{i}<x_{j} \\
0 \text { se } x_{i}=x_{j} \\
-1 \text { se } x_{i}>x_{j}
\end{array}\right.
$$

Se l'ipotesi nulla $\mathrm{H} 0$ è rigettata, il trend è considerato significativo con verso concorde alla pendenza della regressione lineare (Fig. 9).

\section{Bilancio idrico}

Ai fini dello svolgimento della Fase B del Test 1, i vari elementi che compongono il bilancio idrico sono stati calcolati in base ai risultati di un preesistente modello numerico di flusso relativo alla piana d'Aosta (Stefania et al. 2018b) risolto con il codice MODFLOW 2005 (Harbaugh 2005). Questo modello è relativo al periodo 2009-2010. In particolare, i vari elementi che compongono il bilancio sono stati quantificati dal modello, relativamente ai 2 anni considerati, come dettagliato di seguito:

- LTAAQ - calcolato sommando gli emungimenti principali insistenti sull'acquifero, che sono dovuti, nell'area di studio, a 8 pozzi pubblici idropotabili e a 19 pozzi industriali (Stefania et al. 2018b);

- LTAAR - calcolato sommando l'infiltrazione efficace stimata spazialmente su 4 zone diverse (Stefania et al. 2018b) e l'afflusso d'acqua proveniente dai corpi idrici superficiali (Dora Baltea e affluenti) in condizione

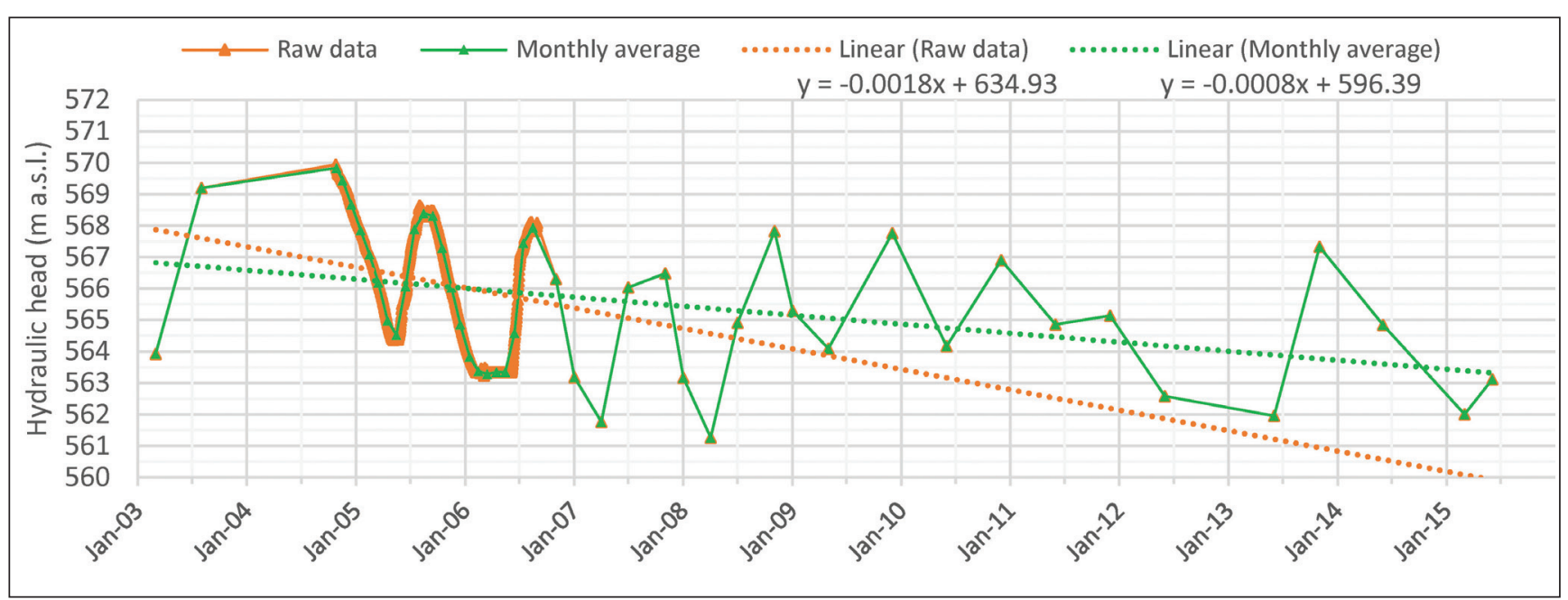

Fig. 8 - Confronto tra le regressioni lineari dei dati grezzi (in arancio) e delle medie mensili (in verde) di un dataset di esempio (pozzo Ao51).

Fig. 8 - Comparison between linear regressions of raw data (in orange) and monthly averaged data (in green) of an example dataset (well Ao51). 


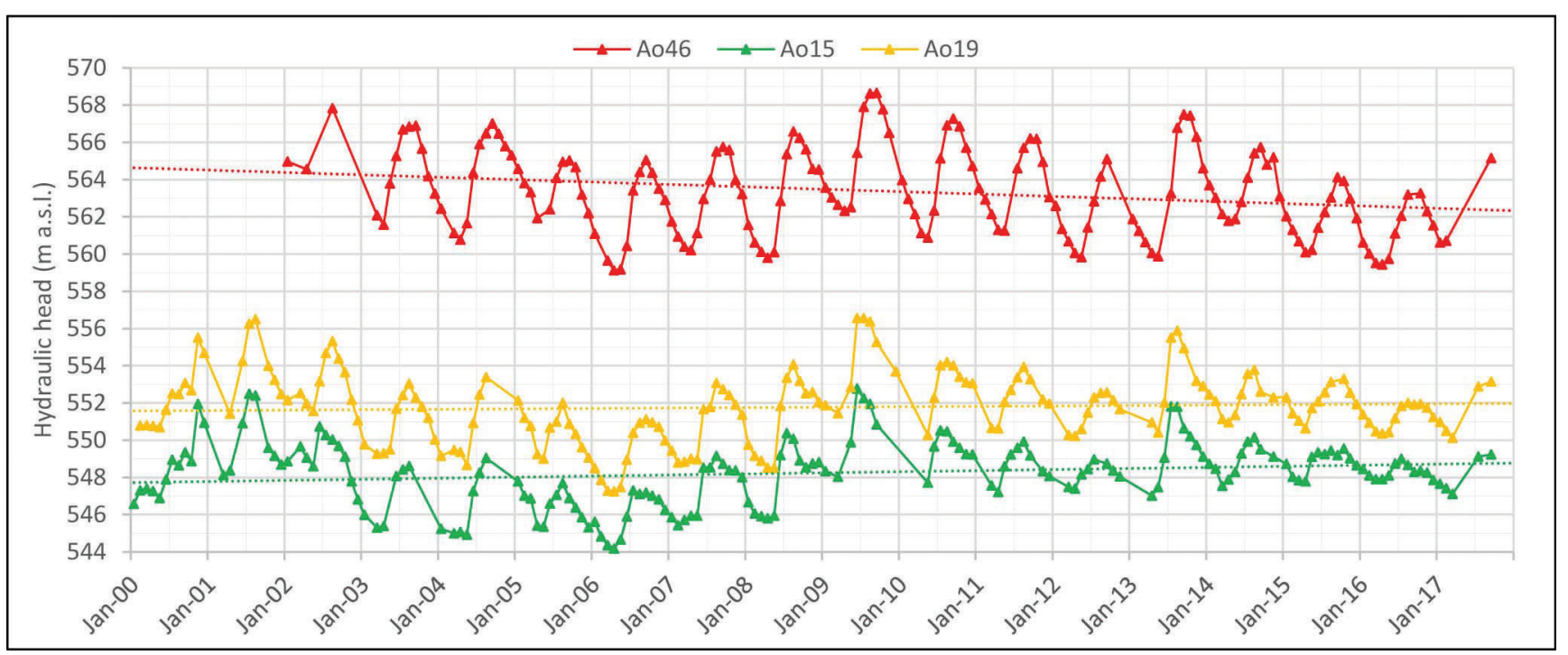

Fig. 9 - Esempi di trend pluriennali nelle serie piezometriche: trend positivo in verde (Ao15), trend negativo in rosso (Ao46), e serie stabile in giallo (Ao19) (si veda la Tab. 1 per i dettagli relativi ai dati di ciascuna stazione di monitoraggio considerata).

Fig. 9 - Example of different multi-year trends in piezometric data series: positive trend in green (Ao15), negative trend in red (A046) and no trend in yellow (Ao19) (see Tab. 1 for details on monitoring points data).

alimentante; i valori di infiltrazione efficace di ciascuna zona sono stati calcolati come surplus idrico del suolo determinato mediante il modello di bilancio idrologico di Thornthwaite-Mather, a partire dalle medie mensili di precipitazione e temperatura misurate sulle stazioni meteoclimatiche presenti nell'area di studio (Bonomi et al. 2013); le portate d'acqua che dai corpi idrici superficiali vanno ad alimentare la falda sono state calcolate tramite il modello numerico di Stefania et al. (2018b), grazie all'uso del pacchetto Streamflow-Routing 2 (SFR2; Niswonger and Prudic 2005) di MODFLOW per la simulazione dei corpi idrici superficiali.

- $\quad$ EFN - calcolato tramite il modello numerico di Stefania et al. (2018b), utilizzando il suddetto pacchetto SFR2 di MODFLOW, come portata di falda drenata dai corpi idrici superficiali.

Così come richiesto dalle linee guida si è proceduto al calcolo del rapporto tra LTAAQ e AGR a cadenza sia annuale che mensile.

\section{Risultati e Discussione Test 1}

La valutazione della tendenza pluriennale dei livelli di falda su singolo pozzo (Fase A) ha evidenziato una situazione generalmente stabile dei livelli di falda, con 49 punti di monitoraggio senza trend significativo, 8 con trend negativo e 7 con trend positivo (Tab. 1). Mappando i risultati (Fig. 10a) è possibile notare come i punti con tendenza negativa si concentrino ad ovest di Aosta e sulla parte sud-orientale della conoide presente, mentre quelli con tendenza positiva si concentrino ad est del capoluogo.

La diminuzione registrata nei piezometri occidentali (Fig. 10b) assume valori di 10-30 cm/anno nel periodo 20022017 circa, ad eccezione di Ao51 la cui serie si ferma al
2015. Sulla conoide di Aosta, in prossimità della confluenza del Torrente Buthier nella Dora Baltea, si ha invece una diminuzione di circa 5-10 cm/anno nel periodo 2008-2017, o 2002-2017 per Ao22 (Fig. 10c). La serie di Sc16 (Fig. 10c), anch'essa in diminuzione, è contraddistinta da una scarsa lunghezza della serie temporale, dal 2010 al 2017, e bassa numerosità campionaria (27 misure totali disponibili nel periodo considerato). Va rilevato che si tratta in ogni caso di diminuzioni di minima entità, specie considerando lo spessore dell'acquifero in questa zona $(70-90 \mathrm{~m})$. Rivolgendo l'attenzione ai piezometri con trend positivo, questi sono maggiormente concentrati ad est di Aosta (Fig. 10c) dove sono registrate serie di durata confrontabile e numerosità di dati simile che mostrano un aumento di circa 10-17 cm/anno. Ao07, ad ovest di Aosta (Fig. 10b), è in controtendenza rispetto ai trend vicini, ma la serie risulta viziata da anni mancanti. Nel territorio di Brissogne, il piezometro Br04 (Fig. 10d) mostra un aumento di 5 cm/anno nel periodo 2005-2015.

Le differenti durate e numerosità campionarie delle serie non consentono un confronto diretto tra di esse per via della differente rappresentatività dei dataset. La risposta del test è fortemente influenzata dalla popolosità dei dataset e, anche in presenza di pendenze simili, i risultati possono variare molto. Inoltre, le pendenze dei trend subiscono variazioni importanti in funzione dell'inclusione delle fluttuazioni pluriennali antecedenti il 2009 e serie riferite a periodi diversi non risultano confrontabili. A causa di queste differenze di composizione campionaria e di significatività statistica e per la mancanza di copertura di alcune aree estese, si è ritenuto che una spazializzazione dei risultati, come suggerito dalle linee guida, non sia applicabile.

I risultati del bilancio idrico, secondo la Fase B del test, sono riportati in Fig. 11. Le barre del grafico esprimono la differenza, calcolata sia a passo mensile sia annuale, tra risorsa 


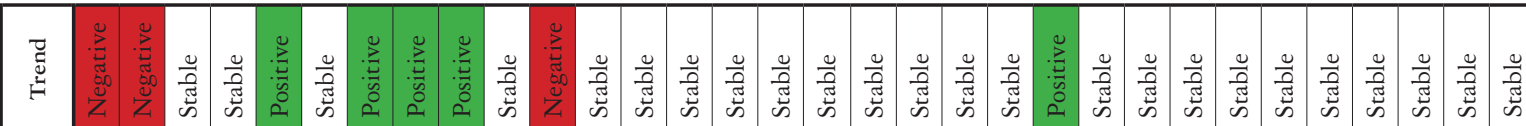

5

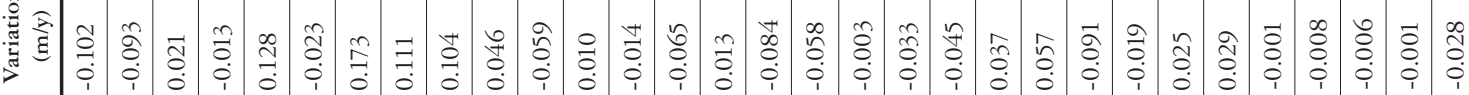

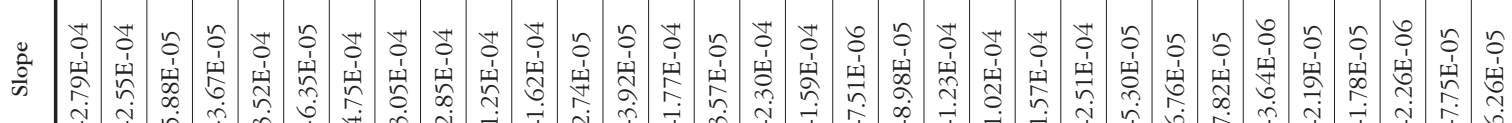

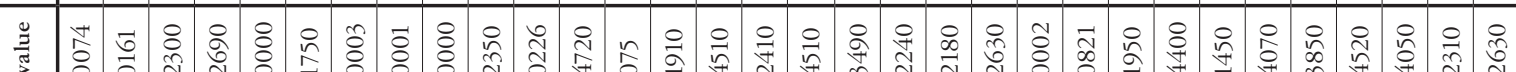

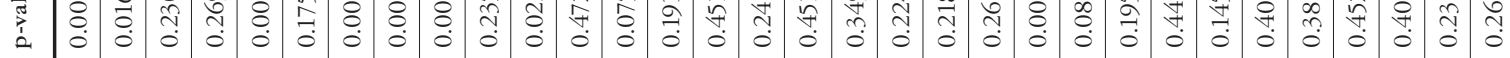

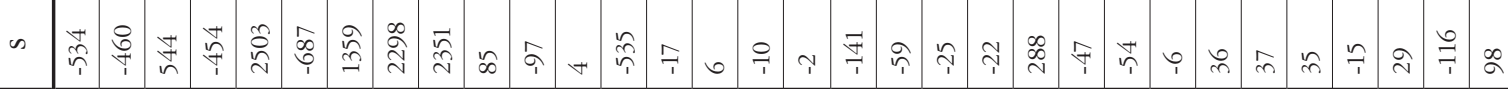

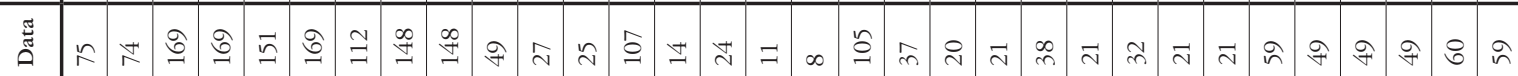

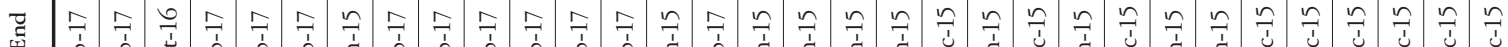

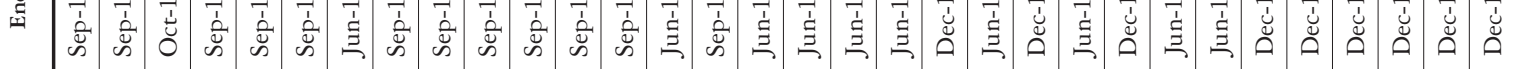

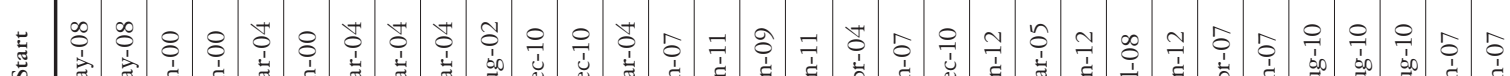

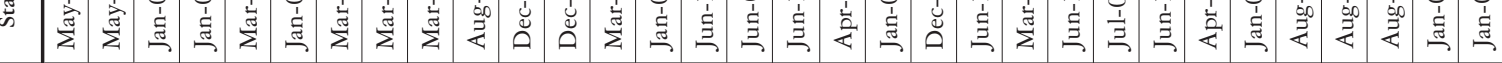

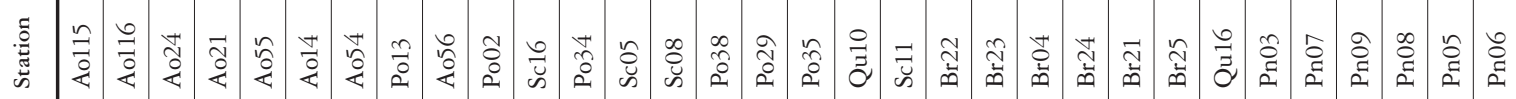

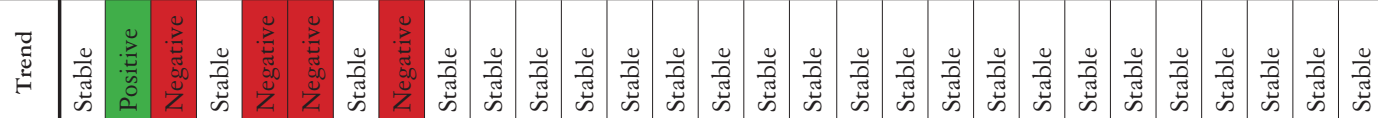

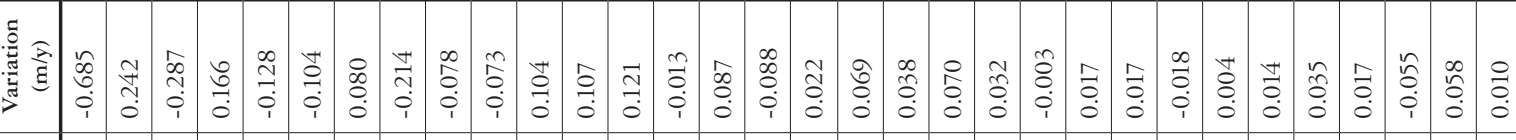

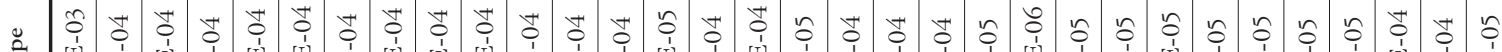

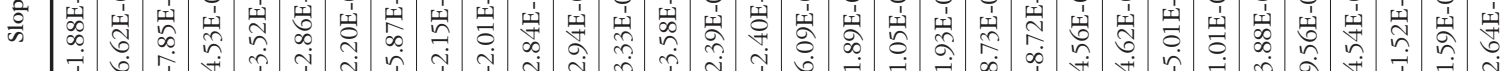
虫

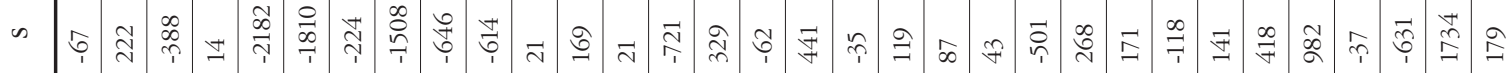

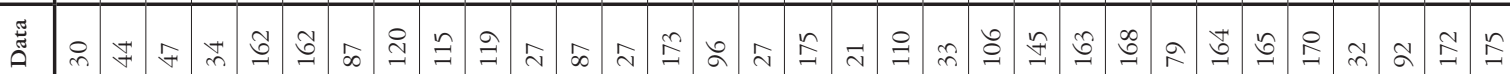

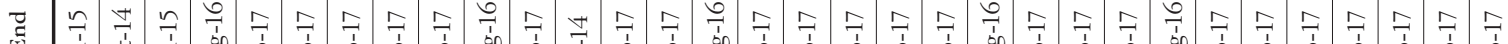

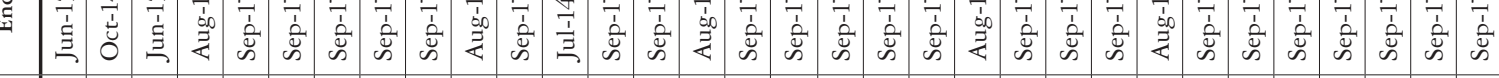
若 至

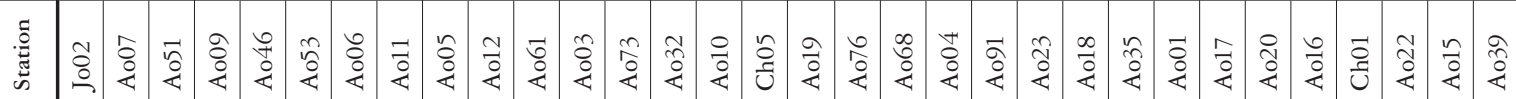



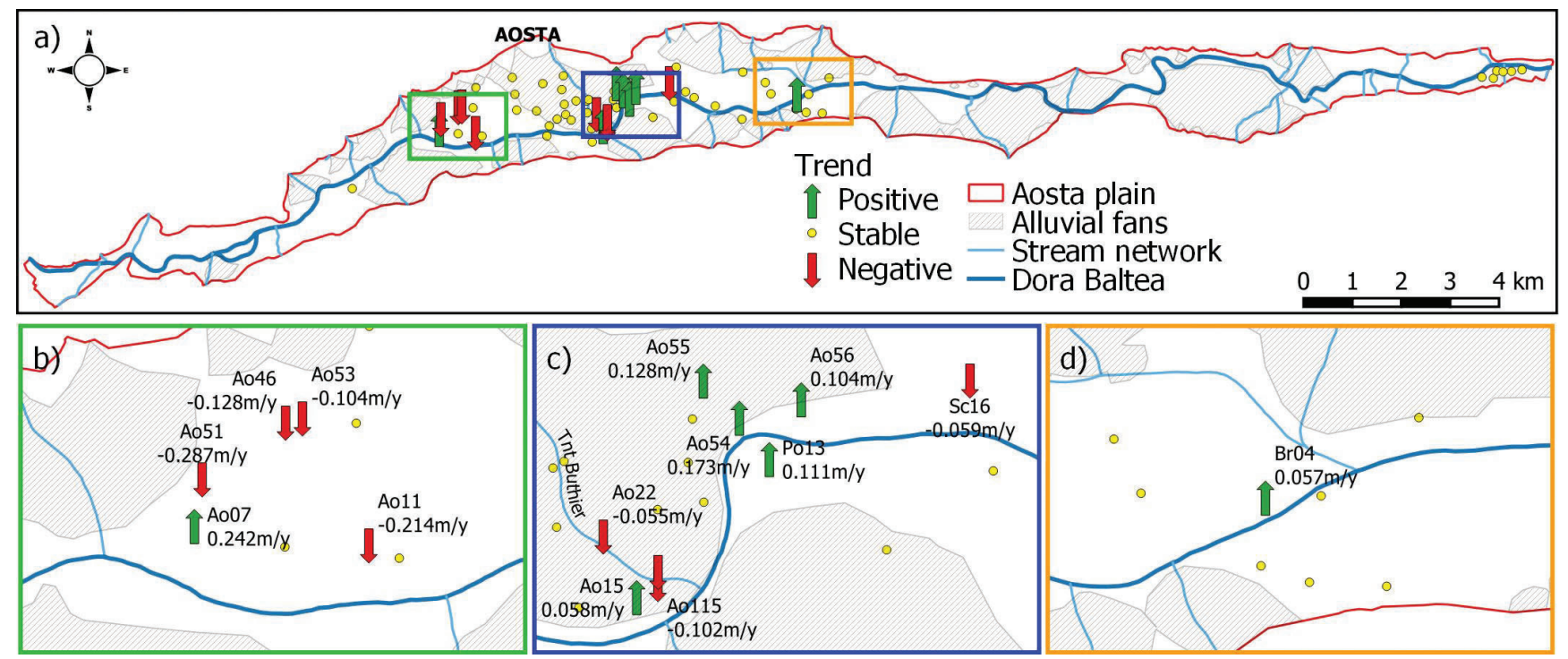

Fig. 10 - Mappa delle tendenze pluriennali calcolate su ciascuna stazione di monitoraggio.

Fig. 10 - Map with multi-year trends calculated for each monitoring station.

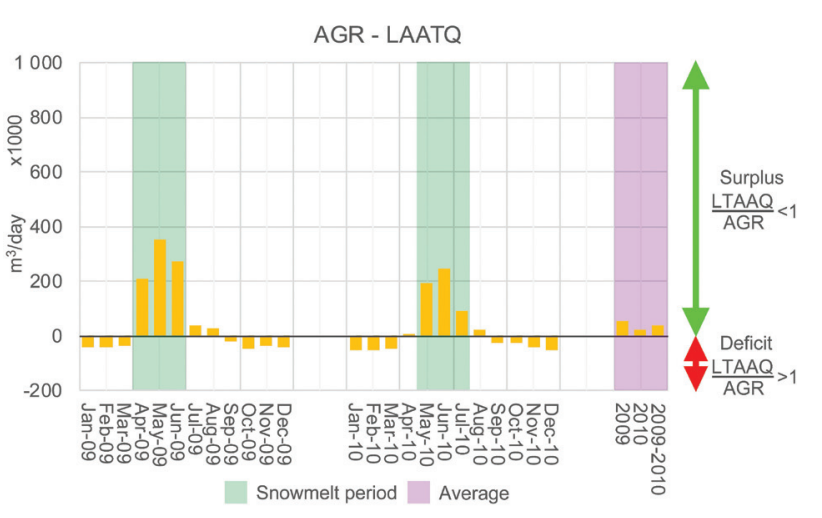

Fig. 11 - Differenza tra risorse idriche disponibili (AGR) e prelievi (LTAAQ) su base mensile, media annua e media bi-annuale.

Fig. 11 - Difference between available groundwater resources (AGR) and well pumping (LTAAQ) on a monthly basis, annual average and bi-annual average.

idrica disponibile (AGR) e prelievi (LTAAQ). Questi ultimi sono ascrivibili per la maggior parte a pochi soggetti (ad es. uso idropotabile del Comune di Aosta, utilizzo industriale di uno stabilimento in Aosta) che misurano regolarmente $\mathrm{i}$ volumi estratti dai propri pozzi.

Analizzando il bilancio a cadenza mensile, è osservabile una diversa risposta del sistema in funzione della stagionalità. In particolare, nei mesi in cui la ricarica è minima o assente (da gennaio a marzo e da settembre a dicembre), i prelievi (LTAAQ) risultano maggiori della risorsa idrica disponibile (AGR) mandando il sistema in deficit idrico (LAATQ/AGR $>1$ ). Nei mesi in cui invece lo scioglimento nivale è massimo (da aprile a giugno nel 2009 e da maggio a luglio nel 2010), la ricarica è sempre di gran lunga superiore al pompaggio mantenendo il sistema in una fase di surplus idrico (LAATQ/AGR $<1$ ).

Analizzando il problema alla risoluzione temporale annuale, scala temporale di riferimento secondo la normativa, emerge che la risorsa idrica disponibile (AGR) è sempre in grado di compensare i prelievi idrici (LAATQ), mantenendo il sistema in una situazione di generale surplus idrico (LAATQ/AGR $<1$ ). Lapplicazione di questo test ha dato un esito positivo, quindi l'acquifero studiato presenta uno stato quantitativo "buono" secondo questo test.

\section{Test 2, Test 3 e Test 4}

Per l'applicazione del Test 2, ci si è avvalsi delle classificazioni qualitative dei corpi idrici superficiali effettuate dall'Autorità di Bacino del Fiume Po (AdbPo 2016) e da ARPA Valle d'Aosta (ARPA VdA 2017). I giudizi di stato elaborati dagli Enti mostrano che le aste fluviali presenti nell'area sono classificate in "buono" stato chimico ed ecologico, ad eccezione di 3 corpi idrici (torrente de Clusellaz, torrente de Comboué e torrente de Verrogne) che sono classificati come corpi idrici altamente modificati a causa delll'antropizzazione dell'alveo e delle derivazioni di acque superficiali (ARPA VdA 2017). Non comportando un ruolo determinate per lo stato "scarso" di questi corpi idrici superficiali, le risorse idriche sotterrannee presenti nell'area di studio sono giudicate in stato "buono" secondo questo test.

In merito al Test 3, per individuare i contesti da valutare è stato fatto riferimento al report sullo stato di conservazione degli ecosistemi elaborato da ISPRA (ISPRA 2016). Questo report mostra ampie zone urbanizzate e criticità legate all'antropizzazione e al rapporto superficie reale/potenziale, non riconducibili alle acque sotterranee. Inoltre, nell'area di studio è presente una zona umida appartenente alla rete Natura 2000, la Zona di Protezione Speciale (ZPS) di Les Iles di Saint-Marcel, che per definizione dipende dalle acque sotterranee. La valutazione di stato per questa ZPS elaborata dalla Regione (Regione Autonoma Valle d'Aosta 2003), indica che esistono criticità legate alla contrazione del biotipo per espansione di strutture antropiche, ma relativamente alle acque, la falda supplisce alle necessità ecologiche. Non essendo 
state riscontrate criticità per gli ecosistemi riconducibili alle acque sotterranee, è attribuibile uno stato quantitativo "buono" secondo questo test.

Per quanto riguarda il Test 4, studi precedenti sul territorio regionale (Rotiroti et al. 2015b; Novel et al. 2002; Tiwari et al. 2017) hanno evidenziato una natura neutro-alcalina delle acque, con un'idrochimica controllata principalmente dai fenomeni di dissoluzione di carbonati, solfati e silicati dai minerali presenti, con basse concentrazioni di cloruri. Escludendo la possibilità di intrusione salina nell'area di studio, lo stato quantitativo è giudicato "buono" secondo questo test.

Alla luce dei risultati ottenuti, in tutti e quattro i test è stato attribuito un giudizio positivo che porta ad assegnare il "buono" stato quantitativo al corpo idrico sotterraneo presente nella piana d'Aosta.

\section{Criticità emerse nei dati a disposizione e raccomandazioni per i monitoraggi futuri}

Lapplicazione del Test 1 ha evidenziato criticità relative all'applicazione dell'analisi delle tendenze pluriennali dei livelli di falda in funzione dei dati a disposizione, in particolare riconducibili alla composizione dei dataset di misure piezometriche che sono stati spesso caratterizzati per i punti non afferenti alla rete di monitoraggio, oggetto di misure occasionali - da discontinuità campionaria, scarsa numerosità e disomogeneità nella frequenza di monitoraggio, nonché da differenze tra i dataset relative alla composizione e ai periodi monitorati. In particolare, analizzando i risultati dei test statistici sulle serie, è emersa una dipendenza delle risposte dalla lunghezza delle serie, dal periodo considerato e dal numero di misure effettuate; affinché i risultati dei test siano confrontabili, tali serie dovrebbero essere di composizione reciprocamente omogenea.

Alla luce di queste problematiche riscontrate, è possibile fornire delle indicazioni per una migliore pianificazione dei

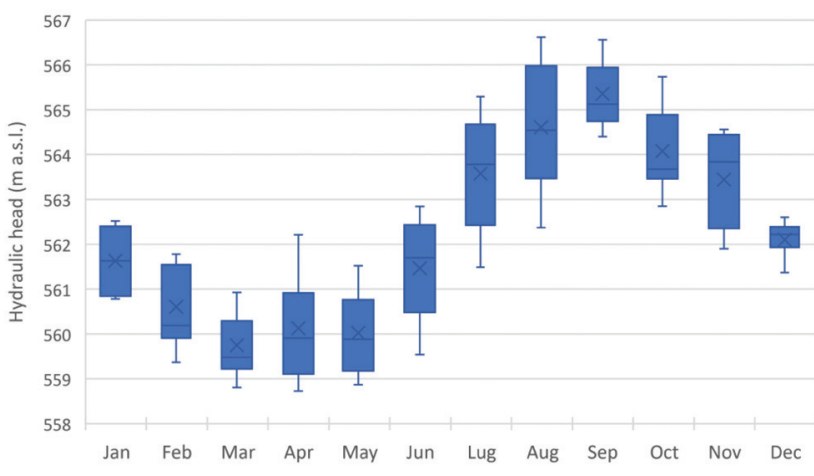

Fig. 12 - Rappresentazione dell'anno medio di riferimento (l'esempio del pozzo Ao12).

Fig. 12 - Representation of average groundwater levels during the year, (the example of well Ao12).

monitoraggi futuri nell'area di studio:

- mantenere l'esistente monitoraggio stagionale costante per consentire di omogeneizzare la composizione delle serie storiche esistenti, affinché queste siano confrontabili;

- $\quad$ integrare nuove stazioni per ottenere una spazializzazione rappresentativa dell'intera area per le future valutazioni di stato quantitativo.

Attraverso l'elaborazione dell'anno medio di riferimento (Fig. 12) per 32 piezometri distribuiti lungo la piana, è possibile individuare i periodi di massima e minima ricarica della falda al fine di determinare i periodi in cui concentrare le misurazioni, per registrare i livelli estremi annui. Spazializzando questi risultati, ad esempio tramite poligoni di Voronoi (Fig. 13), è possibile ottenere una zonazione degli estremi piezometrici, che può supportare la programmazione delle campagne di monitoraggio future. Il minimo appare prettamente primaverile nella zona occidentale, tra marzo e maggio, e si anticipa progressivamente procedendo verso est, verificandosi in febbraio nella zona centro-orientale e in
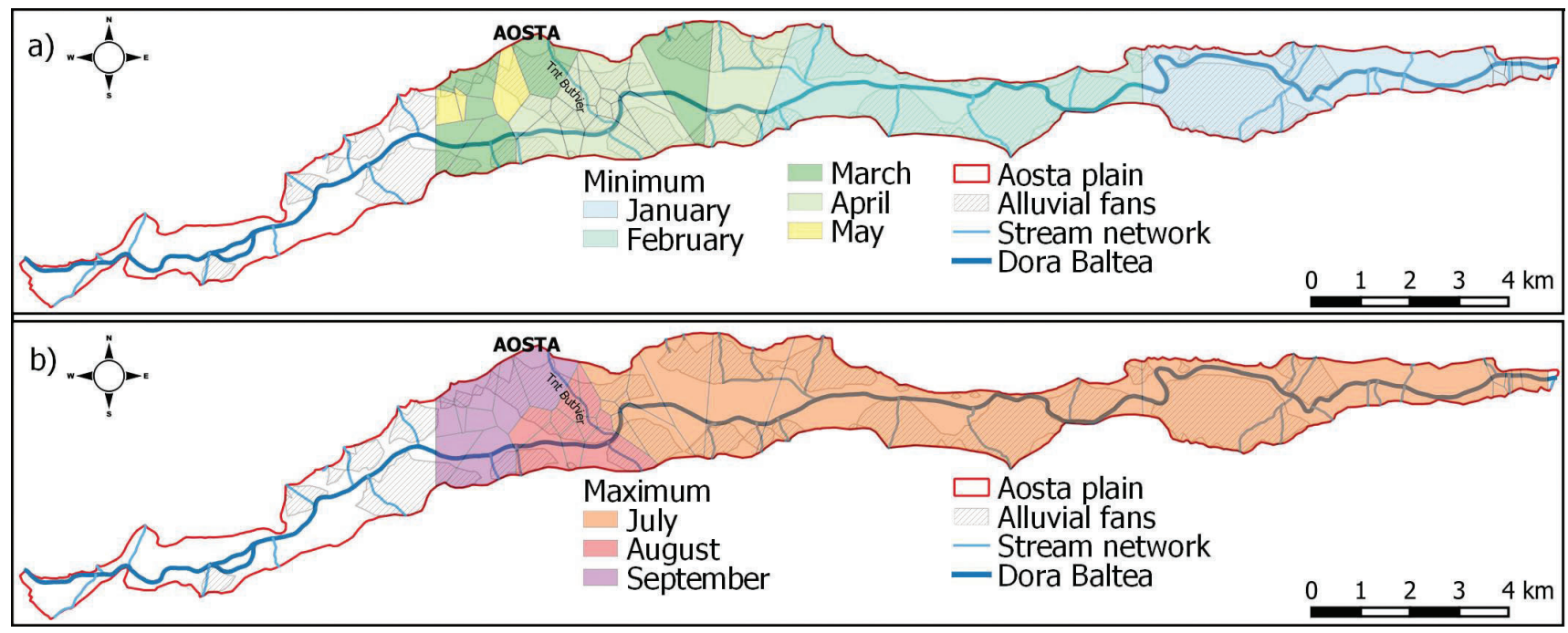

Fig. 13 - Distribuzione spaziale del mese di occorrenza del livello piezometrico minimo (a) e massimo (b) nella piana d'Aosta.

Fig. 13 - Spatial distribution of the occurrence of minimum (a) and maximum (b) groundwater levels in the Aosta Plain. 
gennaio ad est. Anche i massimi mostrano una progressiva anticipazione procedendo longitudinalmente, verificandosi in settembre ad ovest di Aosta, in agosto sulla conoide del torrente Buthier e in luglio nel resto della piana.

\section{Conclusioni}

Le procedure messe in atto hanno permesso di evidenziare difficoltà tecniche ed operative relative al monitoraggio quantitativo dell'acquifero della piana di Aosta, che hanno ostacolato la rigorosa applicazione delle istruzioni fornite dalle linee guida ISPRA. In particolare, per la Fase A del Test 1 relativa alla valutazione delle tendenze pluriennali dei livelli di falda, i requisiti di lunghezza delle serie storiche e di frequenza di monitoraggio richiesti sono risultati non soddisfatti per tutti i punti non afferenti alla rete principale di monitoraggio, rendendo necessario un approccio più tollerante nei confronti delle caratteristiche dei dataset. Ciò ha permesso di valutare più estensivamente le tendenze puntuali, a scapito della significatività statistica. Le differenze nella composizione e lunghezza dei dataset, nei periodi monitorati e nella frequenza di monitoraggio, non hanno permesso un confronto diretto tra le serie storiche e hanno reso poco significativa una loro spazializzazione. Tuttavia, a livello puntuale, l'andamento della falda mostra una generale stabilità, risultato confermato dall'analisi di bilancio idrico che descrive una situazione caratterizzata da brevi deficit invernali più che bilanciati dai surplus estivi. Pertanto, alle acque sotterranee nell'area di studio è attribuibile, ad oggi, un giudizio di stato quantitativo "buono". Va però considerato che i cambiamenti climatici in atto, specie in un'area montana come quella in esame, potrebbero modificare sensibilmente le condizioni attuali nei prossimi anni. Di conseguenza, anche alla luce delle criticità riscontrate dall'applicazione della metodologia ISPRA, risulta necessario non solo mantenere l'attuale struttura del monitoraggio, ma incrementare la rete sia spazialmente che temporalmente per ottenere dataset robusti, aggiornati e uniformi sia in termini di durata che di frequenza di misura, che possano favorire e migliorare le future analisi. La scala temporale di analisi rappresenta un fattore fondamentale, dal momento che le variazioni stagionali possono determinare impatti sulle risorse idriche superficiali e sugli ecosistemi terrestri. Attraverso l'estensione, anche mediante sensori automatici, dell'esistente monitoraggio stagionale sarebbe possibile nei prossimi anni ottenere serie che soddisfino i requisiti posti e riferite a periodi uniformi e sufficientemente lunghi, favorendo un'applicazione rigorosa delle linee guida con risultati più robusti, confrontabili e spazializzabili.

Ringraziamenti: Il presente lavoro è stato finanziato dall'Agenzia Regionale per la Protezione dell'Ambiente della Valle d'Aosta tramite convenzione scientifica n. 2018-CONV25-0025 con l'Università di Milano-Bicocca.

\section{BIBLIOGRAFIA}

AdbPo (2016) Piano di Gestione del distretto idrografico del fiume Po - Aggiornamento 2015, versione marzo 2016 "Po River basin management plan - 2015 update, ver. March 2016". Autorità di bacino del fiume Po, Parma.

ARPA VdA (2017) Classificazione dei corpi idrici superficiali al termine del I PdGPo (2010-2015) "Surface water bodies classification at the end of the I Po River Basin management plan". Available from: http://www.arpa.vda.it/it/acqua/acque-superficiali/acquecorrenti/2771-classificazione-dei-corpi-idrici-superfi-ciali-al-termine-del-i-pdgpo-2010-2015

Boni C, Petitta M, Preziosi E, Sereni M (1993). Genesi e regime di portata delle acque continentali del Lazio "Origin and flow of continental water in Lazio". CNR, Roma.

Bonomi T, Fumagalli L, Benastini V, Rotiroti M, Capodaglio P, Simonetto S (2013) Preliminary groundwater modelling by considering the interaction with superficial water: Aosta plain case (northern Italy). Acque Sotterranee - Italian Journal of Groundwater 2(1): 31-45. doi:10.7343/AS-017-13-0041

Bonomi T, Fumagalli L, Rotiroti M, Bellani A, Cavallin A (2014) The hydrogeological well database TANGRAM $\odot$ : a tool for data processing to support groundwater assessment. Acque Sotterranee Italian Journal of Groundwater 3(2): 35-45. doi:10.7343/AS-07214-0098

Bonomi T, Fumagalli L, Stefania G A, Rotiroti M, Pellicioli F, Simonetto F, Capodaglio P (2015a) Groundwater contamination by $\mathrm{Cr}(\mathrm{VI})$ in the Aosta Plain (northern Italy): characteriztion and preliminary modeling. Rendiconti Online della Società Geologica Italiana 35: 21-24. doi:10.3301/ROL.2015.54

Bonomi T, Fumagalli L, Rotiroti M, Perego R, Simonetto F, Capodaglio P (2015b) Groundwater flow modelling of the Aosta plain in northern Italy. In Lollino G. et al. (ed.) Engineering Geology for Society and Territory - Volume 3: River Basins, Reservoir Sedimentation and Water Resources (p. 227-230). Springer International Publishing, Switzerland. doi:10.1007/978-3-319-09054-2_46

Braca G, Bussettini M, Lastoria B, Mariani S (2013) Linee guida per l'analisi e l'elaborazione statistica di base delle serie storiche di dati idrologici "Guidelines for basic statistical analysis of hydrological time series". ISPRA, Manuali e Linee Guida 84/13.

Brunke M, Gonser T (1997) The ecological significance of exchange processes between rivers and groundwater. Freshwater Biology 37: 1-33. doi:10.1046/j.1365-2427.1997.00143.x

Capodaglio P, Simonetto F (2017a) Recenti indagini geofisiche profonde nella piana di Aosta "Recent deep geophysical investigations in the Aosta Plain”. Geologia dell'Ambiente 2(suppl.): 18-21.

Capodaglio P, Simonetto F (2017b) Il monitoraggio ambientale delle acque sotterranee nella piana di Aosta "Environmental monitoring of groundwater resources in the Aosta Plain". Geologia dell'Ambiente 2(suppl.): 48-50.

D. Lgs. 30/09 (2009) Decreto Legislativo n. 30 del 16 marzo 2009 sull'attuazione della direttiva 2006/118/CE relativa alla protezione delle acque sotterranee dall'inquinamento e dal deterioramento "Legislative Decree on the implementation of Directive 2006/118/EC on the Protection of Groundwater from Contamination and Degradation".

D. Lgs. 152/99 (1999) Decreto Legislativo n. 152 del 11 maggio 1999 recante disposizioni sulla tutela delle acque dall'inquinamento e recepimento della direttiva 91/271/CEE concernente il trattamento delle acque reflue urbane e della direttiva 91/676/CEE relativa alla protezione delle acque dall'inquinamento provocato dai nitrati provenienti da fonti agricole "Legislative Decree on the protection of water resources from pollution and on the implementation of Directive 91/271/ EEC concerning urban waste water treatment and Directive 91/676/EEC concerning the protection of waters against pollution caused by nitrates from agricultural sources".

DM 260/2010 (2010) Decreto Ministeriale n. 260 del 8 novembre 2010 recante i criteri tecnici per la classificazione dello stato dei corpi idrici superficiali "Ministerial Decree on technical criteria for the classification of the status of surface water bodies". 
EC (2000) Directive 2000/60/EC of 23 October 2000 establishing a framework for Community action in the field of water policy. Water Framework Directive - WFD. European Parliament and Council.

EC (2006) Directive 2006/118/EC of 12 December 2006 on the protection of the groundwater against pollution and deterioration. Groundwater Directive - GWD. European Parliament and Council.

EC (2009) CIS Guidance Document No.18. Guidance on Groundwater Status and Trend Assessment. Technical Report No.026-2009.

EC (2011). CIS Guidance Document No.06. Technical Report on Groundwater Dependent Terrestrial Ecosystems. Technical Report No.06-2011.

Guerra M, Preziosi E, Ghergo S, Calace N, Guyennon N, Marcaccio M, Menichetti S, Romano E (2017) Linee guida per la valutazione delle tendene ascendenti e d'inversione degli inquinanti nelle acque sotterranee (DM 6 luglio 2016) "Guidelines for the identification of any significant and sustained upward trends in pollutant concentrations and for the definition of the starting point for trend reversal in groundwater". ISPRA, Manuali e Linee Guida 161/2017.

Harbaugh A W (2005) MODFLOW-2005, The U.S. Geological Survey modular ground-water model: the ground-water flow process. U. S. Geol. Surv. Techniques and Methods 253

Hatton T J, Evans R (1998) Dependence of ecosystems on groundwater and its significance to Australia. LWRRDC Occasional Paper No 12/98. Available at https://library.dbca.wa.gov.au/static/FullTextFiles/018743.pdf

Huh S, Dickey D A, Meador M R, Ruh K E (2005) Temporal analysis of the frequency and duration of low and high streamflow: years of record needed to charaterize streamflow variability. Journal of $\mathrm{Hy}$ drology 310: 78-94. doi:10.1016/j.jhydrol.2004.12.008

ISPRA (2016) Mappatura degli ecosistemi e valutazione del loro stato di conservazione "Ecosystem mapping and conservation status assessment". Strategia Nazionale per la Biodiversità, available at: https:// ftp.minambiente.it/pnm/Strategia_Nazionale_Biodiversita/Capitale_Naturale/Mappatura\%20Ecosistemi_MAES/VALLE\%20D_ AOSTA/

Mann H B (1945) Nonparametric tests against trend. Econometrica 13: 245-259. doi:10.2307/1907187

Niswonger R G, Prudic D E (2005) Documentation of the Streamflow-Routing (SFR2) Package to include unsaturated flow beneath streams: a modification to SFR1. U. S. Geol. Surv. Techniques and Methods 6-A13, USGS, Reston, VA.

Novel J P, Puig J M, Zuppi G M, Dray M, Dzikowski M, Jusserand C, Money E, Nicoud G, Parriaux A, Pollicini F (2002) Complexité des circulations dans l'aquifere alluvial de la plaine d'Aoste (Italie): Mise en évidence par l'hydrogéochimie "Complexity of the groundwater flow in the alluvial aquifer of the Aosta plain (Italy): Study with bydrogeochemistry". Eclogae Geologicae Helvetiae 95(3): 323-331. doi:10.5169/seals-168963

Percopo C, Brandolin D, Canepa M, Capodaglio P, Cipriano G, Gafa R M, Marcaccio M, Mazzola M, Mottola M, Sesia E, Testa M (2017) Criteri tecnici per l'analisi dello stato quantitativo e il monitoraggio dei corpi idrici sotterranei "Technical criteria for quantitative status assessment and monitoring of groundwater bodies". ISPRA, Manuali e Linee Guida 157/2017.
Regione Autonoma Valle d'Aosta (2003) Zona umida di Les Iles di Saint-Marcel - IT1205070, Formulario standard per Zone di Protezione Speciale (ZPS) "Les Iles of Saint-Marcel wetland-IT1205070, Standard form for Special Protection Area (SPA)". Available at: http:// www.regione.vda.it/risorsenaturali/conservazione/natura2000/siti/ IT1205070/default_i.aspx

Rotiroti M, Di Mauro B, Fumagalli L, Bonomi T (2015a) COMPSEC, a new tool to derive natural background levels by the component separation approach: application in two different hydrogeological context in northern Italy. Journal of Geochemical Exploration 158: 44-54. doi:10.1016/j.gexplo.2015.06.017

Rotiroti M, Fumagalli L, Frigerio M C, Stefania G A, Simonetto F, Capodaglio P, Bonomi T (2015b) Natural background levels and threshold values of selected species in the alluvial aquifers in the Aosta Valley Region (N Italy). Rendiconti Online della Società Geologica Italiana 35: 256-259. doi:10.3301/ROL.2015.114

Stefania G A, Rotiroti M, Fumagalli L, Zanotti C, Bonomi T (2018a) Numerical modeling of Remediation Scenarios of a Groundwater $\mathrm{Cr}(\mathrm{VI})$ Plume in an Alpine Valley Aquifer. Geosciences 8. doi:10.3390/geosciences8060209

Stefania G A, Rotiroti M, Fumagalli L, Simonetto F, Capodaglio P, Zanotti C, Bonomi T (2018b) Modeling groundwater/surface-water interactions in an Alpine valley (the Aosta Plain, NW Italy): the effect of groundwater abstraction on surface-water resources. Hydrogeology Journal 26: 147-162. doi:10.1007/s10040-017-1633-x

Stefania G A, Rotiroti M, Buerge I J, Zanotti C, Nava V, Leoni B, Fumagalli L, Bonomi T (2019) Identification of groundwater pollution sources in a landfill site using artificial sweeteners, multivariate analysis and transport modeling. Waste Management 95: 116-128. doi:10.1016/j.wasman.2019.06.010

Tiwari A K, Ghione R, De Maio M, Lavy M (2017) Evaluation of hydrogeochemical processes and groundwater quality for suitability of drinking and irrigation purposes: a case study in the Aosta Valley region, Italy. Arabian Journal of Geosciences. doi:10.1007/ s12517-017-3031-z

Tiwari A K, Nota N, Marchionatti F, De Maio M (2017) Groundwater-level risk assessment by using statistical and geographic information system (GIS) techniques: a case study in the Aosta Valley region, Italy. Geomatics, Natural Hazards and Risk 8(2): $1396-$ 1406. doi:10.1080/19475705.2017.1337655.

Triganon A, Dzikowsky M, Navel J P, Dray M, Zuppi G M, Parriaux A (2003) Aquifer-river exchanges in an alpine valley: Quantification and modelling (Valee D'Aoste, Italy). Canadian Journal of Earth Sciences 40: 775-786. doi:10.1139/e03-017

U.S. EPA (2015). ProUCL Version 5.1.002 Technical Guide. Statistical Software for Environmental Applications for Data Sets with and without Nondetect Observations. Available at: https://www. epa.gov/land-research/proucl-software

Winter T M, Harvey J W, Lehn Franke O, Alley W M (1998) Ground Water and Surface Water: a single resource. U.S. Geological Survey Circular 1139

Yue S, Pilon P (2004) A comparison of the power of the t test, MannKendall and bootstrap tests for trend detection. Hydrological Science Journal 49(1): 21-37. doi: 10.1623/hysj.49.1.21.53996 